\title{
Solution-based synthesis of GeTe octahedra at low temperature
}

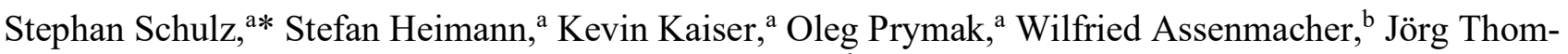 \\ as Brüggemann, ${ }^{\mathrm{c}}$ Bert Mallick, ${ }^{\mathrm{c}}$ Anja-Verena Mudring ${ }^{\mathrm{c}, \mathrm{d}}$ \\ ${ }^{a}$ Institute of Inorganic Chemistry and Center for Nanointegration Duisburg-Essen (CENIDE), University of Duisburg-Essen,

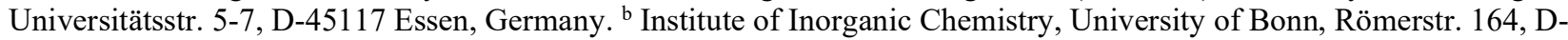 \\ 53117 Bonn, Germany. Inorganic Chemistry III-Materials Engineering and Characterization, Ruhr-University Bochum, \\ 44780 Bochum, Germany. ${ }^{\mathrm{d}}$ Materials Science and Engineering, Iowa State University, Ames, IA 50010, USA.
}

KEYWORDS. Nanostructures - Germanium telluride - Single Crystal and Powder X-Ray Diffraction - TEM

Supporting Information Placeholder

\begin{abstract}
GeTe octahedra were prepared by reaction of equimolar amounts of $\mathrm{GeCl}_{2}$.dioxane and $\mathrm{Te}\left(\mathrm{SiEt}_{3}\right)_{2}$ in oleylamine, whereas a slight excess of the Te-precursor yielded GeTe octahedra decorated with elemental Te nanowires, which can be removed by washing with TOP. The mechanism of the GeTe formation is strongly influenced by the solvent. The expected elimination of $\mathrm{Et}_{3} \mathrm{SiCl}$ (dehalosilylation) only occurred in aprotic solvents, whereas $\mathrm{Te}\left(\mathrm{SiEt}_{3}\right)_{2}$ was found to react with primary and secondary amines with formation of silylamines. Temperaturedependent studies on the reaction in oleylamine showed that crystalline GeTe particles are formed at temperatures higher than $140{ }^{\circ} \mathrm{C} . \mathrm{XRD}$, SAED and HRTEM studies proved the formation of rhombohedral GeTe nanoparticles. These findings were confirmed by a single crystal and powder X-ray analysis. The rhombohedral structure modification was found and the structure was solved in the acentric space group R3m.
\end{abstract}

\section{Introduction}

Nanostructured metal chalcogenides have received increasing interest over the last decade due to their possible technical applications in energy devices such as fuel and solar cells, lightemitting diodes, Li-ion batteries, and thermoelectric devices. ${ }^{[1]}$ Germanium telluride, GeTe, as well as the related GST compounds $\left(\mathrm{Ge}_{2} \mathrm{Sb}_{2} \mathrm{Te}_{5}\right)$ are interesting phase change materials (PCM). ${ }^{[2-6]}$ GST compounds typically crystallize either in a metastable face centered cubic $(\mathrm{C})$ phase or in the stable rhombohedral $(\mathrm{R})$ or hexagonal $(\mathrm{H})$ phase. For materials belonging to these classes frequently reversible amorphous-to-crystalline phase transitions are observed, ${ }^{[7-12]}$ which can be triggered thermally or electrically. Upon crystallization, the conductivity and reflectivity of GeTe significantly changes, hence rendering this material a very promising candidate in non-volatile phase change random access memory (PCRAM) such as CDs or DVDs. ${ }^{[13,14]}$ The ferroelectric phase change of GeTe results from a distinctive structural change between the nonpolar rock salt structure to the polar rhombohedral phase. This symmetry-breaking structural distortion occurs below $350{ }^{\circ} \mathrm{C} .{ }^{[15]}$ Furthermore, GeTe is well known to exhibit thermoelectric properties. ${ }^{[16]}$

Nanosized PCM materials such as GeTe nanoparticles are very interesting because of the well-known influence of the reduced dimensionality of a given material on its physical properties such as melting points or crystallization properties. ${ }^{[17-22]}$ Since GeTe nanoparticles are expected to improve device performances, ${ }^{[23]}$ the interest in suitable precursors for the size- and shape-selective synthesis of GeTe nanocrystals has remarkably increased in recent years and several general reaction pathways have been established. ${ }^{[24]}$ GeTe nanowires containing a large excess of elemental tellurium were formed in high-boiling organic solvents via seedmediated growth using Bi nanoparticles, ${ }^{[25]}$ whereas GeTe nanowires with uniform diameter distributions were grown on the basis of the VLS mechanism. ${ }^{[26]}$ Wet chemical (colloidal) routes also gave access to amorphous and crystalline GeTe nanoparticles with distinctive shape and size. Reactions of $\mathrm{GeI}_{2}$ with TOPTe in the presence of TOP, TOPO and dodecanethiol at $250{ }^{\circ} \mathrm{C}$ yielded amorphous GeTe nanoparticles, whose sizes ranged from 1.7 to 5 $\mathrm{nm} .{ }^{[23]}$ A strong correlation between the crystal size and the crystallization temperature was observed, with a decreasing particle size leading to an increase in crystallization temperature. While for bulk GeTe an amorphous-to-crystalline phase transition was observed at about $170{ }^{\circ} \mathrm{C},{ }^{[27]} 1.8 \mathrm{~nm}$ sized GeTe nanoparticles started to crystallize not before $240^{\circ} \mathrm{C} .{ }^{[23]}$ Crystalline GeTe nanoparticles were also obtained from the reaction of $\mathrm{Ge}\left[\mathrm{N}\left(\mathrm{SiMe}_{3}\right)_{2}\right]_{2}$ and TOPTe in 1 -octadecene at $200{ }^{\circ} \mathrm{C}$ in the presence of oleylamine and dodecanethiol, whereas amorphous GeTe particles were formed in the absence of dodecanethiol at reaction temperatures below $170{ }^{\circ} \mathrm{C} .{ }^{[27]}$ Increasing reaction times produced larger, polydisperse nanoparticles $(9.0-27.8 \mathrm{~nm})$. Alivisatos et al. recently reported on the synthesis of GeTe nanocrystals with variable sizes using germanium precursors of different reactivity. ${ }^{[28]}$ The reaction of $\mathrm{Ge}\left[\mathrm{N}\left(\mathrm{SiMe}_{3}\right)_{2}\right]_{2}$ with TOPTe in 1-dodecanethiol and an excess of TOP at $250{ }^{\circ} \mathrm{C}$ yielded crystalline GeTe nanoparticles with an average size of almost $9 \mathrm{~nm}$, whereas nanoparticles with an average diameter of roughly $17 \mathrm{~nm}$ were obtained at 250 ${ }^{\circ} \mathrm{C}$ in the presence of oleylamine. ${ }^{[29]}$ In contrast, the reaction of the less reactive $\mathrm{GeCl}_{2}$-dioxane with TOPTe and 1-dodecanethiol at $180{ }^{\circ} \mathrm{C}$ produced significantly larger GeTe nanocrystals $(\approx 100$ $\mathrm{nm})$. Micron-sized crystalline GeTe nanoparticles of octahedral shape with terminal $\{111\}$ facets decorated with vertically aligned arrays of Te nanowires were synthesized by reaction of $\mathrm{Ph}_{2} \mathrm{Ge}$ and TOPTe under supercritical conditions in hexane $\left(460{ }^{\circ} \mathrm{C}, 13.6\right.$ $\mathrm{MPa}){ }^{[30]}$ In contrast, GeTe cubes with an average edge length of $1.0 \mu \mathrm{m}$ were obtained from reactions of $\mathrm{GeI}_{2}$ with tert-Buylaminboran $(\mathrm{TBAB})$ in the presence of TOPTe at $180{ }^{\circ} \mathrm{C}^{[31]} \mathrm{In}$ this 
reaction, $\mathrm{GeI}_{2}$ is first reduced to elemental $\mathrm{Ge}^{0}$, which is then consequently oxidized by TOPTe to give GeTe and TOP.

While these synthetic procedures used TOPTe without exception as Te-precursor, we became interested in alternate Teprecursor for the low-temperature synthesis of Te-based materials. Bis(trialkylsilyl)telluranes have been identified as powerful Tetransfer reagents in metal organic chemistry for the synthesis of Te-containing main group and transition metal complexes. ${ }^{[32-37]}$ Moreover, they have recently been used in ALD deposition of Tebased materials films such as $\mathrm{Sb}_{2} \mathrm{Te}_{3}{ }^{\left[{ }^{[38-40]}\right.}$ While this work was under progress, $\mathrm{Kim}$ et al. reported on the time-dependent reaction of $\mathrm{GeCl}_{2} \cdot$ dioxane with $\mathrm{Te}\left(\mathrm{SiEt}_{3}\right)_{2}$ in TOP/TOPO at $250{ }^{\circ} \mathrm{C}$, yielding GeTe nanoparticles of different sizes by a ligand exchange reaction.[41] While short reaction times $(<10 \mathrm{~min})$ yielded amorphous powders, elongated reaction times $(30,60 \mathrm{~min})$ gave faceted, almost monodisperse GeTe cubes ( $350 \mathrm{~nm}$ size). In addition, Buck et al. reported very recently on the polymer-assisted synthesis of GeTe nanoparticles of octahedral facetted shape. ${ }^{[42]}$

We now report herein our detailed studies on the reaction of $\mathrm{GeCl}_{2} \cdot$ dioxane with $\mathrm{Te}\left(\mathrm{SiEt}_{3}\right)_{2}$ in the absence of any reducing agent. The role of the solvent and the influence of reaction time and temperature were studied in detail. In addition, the single crystal X-ray analysis of $\alpha$-GeTe is reported.

\section{Experimental Section}

Materials. $\mathrm{GeCl}_{2}$-dioxane (ABCR) was used as received, 1,3diisopropylbenzene (DIPB, Sigma-Aldrich) was carefully dried over $\mathrm{Na} / \mathrm{K}$ alloy and oleylamine (OA, Acros), hexadecylamine (HDA, Acros), 1-hexadecanethiol (HDT, Sigma-Aldrich), tri-noctylphosphine (TOP, Sigma-Aldrich) and tri-n-octylamine (TOA, Acros) were degassed prior to use. $\mathrm{Te}\left(\mathrm{SiEt}_{3}\right)_{2}$ was synthesized according to literature method. ${ }^{[43]}$ All syntheses were performed under argon atmosphere using standard Schlenk techniques.

Synthesis of GeTe particles. $\mathrm{GeCl}_{2} \cdot$ dioxane $(150 \mathrm{mg}, 0.65$ $\mathrm{mmol}$ ) was suspended in $20 \mathrm{~mL}$ of the solvent (oleylamine, hexadecylamine, 1-hexadecanethiol, DIPB, tri-n-octylamine) and heated to $150{ }^{\circ} \mathrm{C}$. Te$\left(\mathrm{SiEt}_{3}\right)_{2}(200 \mathrm{mg}, 0.56 \mathrm{mmol})$ was injected into the yellow suspension, immediately giving a black suspension that was stirred for additional $4 \mathrm{~h}$. The reaction mixture was allowed to cool down to ambient temperature and the GeTe particles were isolated by centrifugation, repeatedly washed (three times) with $\mathrm{CHCl}_{3}$ and dried under vacuum.

Thermal Analysis. TGA/DTA was performed on a Mettler Toledo Star 1 machine under Ar flow (heating rate $2{ }^{\circ} \mathrm{C} \mathrm{min}^{-1}$ ).

Single Crystal X-ray Diffraction. Single crystal X-ray analysis was performed on a Stoe IPDS I diffractometer (Mo $\mathrm{K}_{\alpha}$ radiation, $\lambda=0.71073 \AA$ ). Crystallographic data are summarized in Table 1. Data were collected at 80(2) and 298(2) K. The structure was solved by Direct Methods (SIR-92) and refined anisotropically by full-matrix least-squares on $\mathrm{F}^{2}$ (SHELXL-97). ${ }^{[44,45]}$ Absorption corrections were performed semi-empirically by approximating the true crystal size and shape from equivalent reflections (Stoe X-Shape).

X-ray Powder Diffraction (XRD). XRD patterns were obtained at ambient temperature, i.e. at $25 \pm 2{ }^{\circ} \mathrm{C}$, using a Bruker D8 Advance powder diffractometer in Bragg-Brentano mode with $\mathrm{Cu}$ $\mathrm{K}_{\alpha}$ radiation $(\lambda=1.5418 \AA, 40 \mathrm{kV}$ and $40 \mathrm{~mA})$. The powder samples were investigated in the range of 5 to $90^{\circ} 2 \theta$ with a step size of $0.01^{\circ} 2 \theta$ and a counting time of $0.3 \mathrm{~s}$. For each Rietveld refinement, performed with the program package TOPAS 4.2 from Bruker, the instrumental correction as determined with a standard powder sample $\mathrm{LaB}_{6}$ from NIST (National Institute of
Standards and Technology) as standard reference material (SRM 660b) was taken into account.

SEM Analysis. SEM studies were carried out on an ESEM Quanta 400 FEG and a Jeol JSM 6510 equipped with an energy dispersive X-ray spectroscopy (EDX) device (Bruker Quantax 400).

TEM Analysis. Transmission electron microscopy (TEM) studies were performed on a Philips CM300UT-FEG operated at 300 $\mathrm{keV}$ and equipped with a germanium EDS-detector (ThermoScientific NSS6) for EDS-analysis. The samples were prepared on perforated carbon foils without further grinding.

\section{Results and Discussion}

The reaction of $\mathrm{GeCl}_{2} \cdot$ dioxane with $\mathrm{Te}\left(\mathrm{SiEt}_{3}\right)_{2}$ was monitored by ${ }^{1} \mathrm{H}$ and ${ }^{13} \mathrm{C}$ NMR spectroscopy (fig. 1 SI). Temperaturedependent spectra, which were recorded in $\mathrm{C}_{6} \mathrm{D}_{6}$ and in THF- $\mathrm{d}_{8}$ solutions, clearly showed the formation of $\mathrm{Et}_{3} \mathrm{SiCl}$ starting at 25 ${ }^{\circ} \mathrm{C}$. Comparable findings were reported by Leskelä et al. for the ALD deposition of metal chalcogenide thin films by reaction of $\mathrm{Te}\left(\mathrm{SiEt}_{3}\right)_{2}$ and metal halides such as $\mathrm{SbCl}_{3}$ and $\mathrm{GeCl}_{2}$.dioxane, respectively. ${ }^{[38,39,46]}$

$$
\mathrm{GeCl}_{2} \cdot \text { dioxane }+\mathrm{Te}\left(\mathrm{SiEt}_{3}\right)_{2} \longrightarrow \mathrm{GeTe}+2 \mathrm{Et}_{3} \mathrm{SiCl}
$$

Scheme 1. Reaction of $\mathrm{GeCl}_{2}$-dioxane and $\mathrm{Te}\left(\mathrm{SiEt}_{3}\right)_{2}$ in $\mathrm{C}_{6} \mathrm{D}_{6}$ and THF- $\mathrm{d}_{8}$.

In contrast, the reaction of $\mathrm{GeCl}_{2} \cdot$ dioxane and $\mathrm{Te}\left(\mathrm{SiEt}_{3}\right)_{2}$ in oleylamine did not proceed with elimination of $\mathrm{Et}_{3} \mathrm{SiCl}$ (fig. $2 \mathrm{SI}$ ). Oleylamine is known to serve as capping and reducing agent[47] and Alivisatos et al. reported on reactions of $\mathrm{GeCl}_{2} \cdot$ dioxane and $\mathrm{Ge}\left[\mathrm{N}\left(\mathrm{SiMe}_{3}\right)_{2}\right]_{2}$ with primary amines (oleylamine) and alkanethiols (1-dodecanethiol), which proceeded at elevated temperatures with subsequent formation of $\mathrm{Ge}(0)$ nanoparticles. ${ }^{[29]}$ The lower reduction rate of $\mathrm{GeCl}_{2}$. dioxane compared to $\mathrm{Ge}\left[\mathrm{N}\left(\mathrm{SiMe}_{3}\right)_{2}\right]_{2}$ resulted in lower $\mathrm{Ge}(0)$ particle nucleation rates and increasing nanoparticle sizes. However, we proved by ${ }^{1} \mathrm{H}$ NMR spectroscopy (fig. $2 \mathrm{SI}$ ) that oleylamine reacts immediately with $\mathrm{Te}\left(\mathrm{SiEt}_{3}\right)_{2}$ at ambient temperature with formation of silylamine (oleylN(H)SiEt 3 ) and subsequent formation of a deep-red solution, which is characteristic for the formation of tellurium polyanions such as the $\left[\mathrm{Te}_{4}\right]^{2-}$ dianion. ${ }^{[48]}$ According to these findings, we assume that in situ formed $\mathrm{Te}-\mathrm{H}$ species, which are strong acids, are deprotonated by the excess amine base. Tellurium polyanions then react with $\mathrm{GeCl}_{2}$.dioxane with formation of GeTe. According to these studies, oleylamine has to be considered rather as reagent that has a strong influence on the whole reaction mechanism, than as simple capping agent.

Reactions of $\mathrm{Te}\left(\mathrm{SiEt}_{3}\right)_{2}$ and $\mathrm{GeCl}_{2} \cdot$ dioxane were investigated in non-coordinating, aprotic (DIPB) and in coordinating, protic solvents (oleylamine) at $160{ }^{\circ} \mathrm{C}$ in order to further investigate the role of the solvent on the particle composition and morphology. 

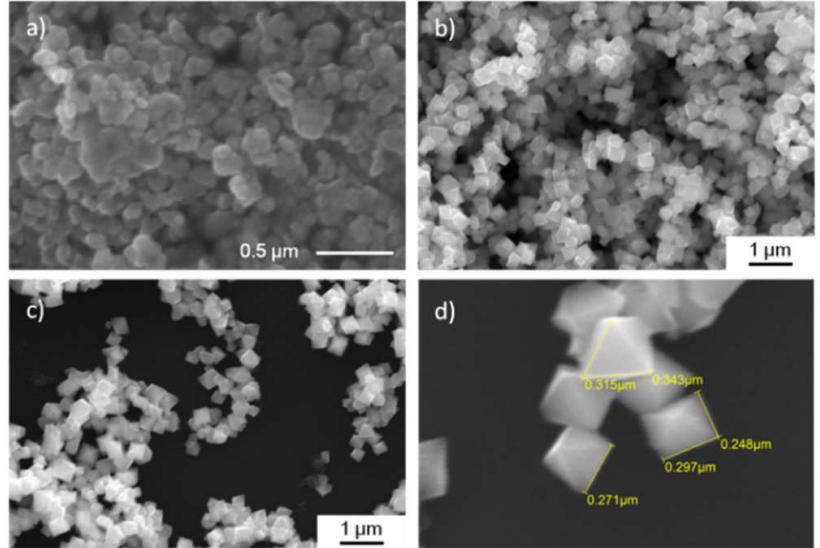

Figure 1. SEM micrographs of octahedral GeTe microcrystallites as-obtained in DIPB (a) and in oleylamine (b), c), d)) at $160^{\circ} \mathrm{C}$.

SEM images of the resulting materials showed the formation of largely agglomerated, non-crystalline particles in DIPB, whereas GeTe particles of octahedral shape were obtained in oleylamine (Fig. 1). The elemental composition (Ge, Te) in both materials are in accordance with the formation of pure GeTe. The edge lengths of the GeTe octahedra ranged from 250 to $350 \mathrm{~nm}$. This is significantly smaller compared to the octahedral GeTe crystallites synthesized in supercritical hexane at significantly higher reaction temperatures $\left(460{ }^{\circ} \mathrm{C}\right)$, which showed edge lengths of about 1 $\mu \mathrm{m} .{ }^{[30]}$ Moreover, these octahedra were largely decorated with elemental tellurium nanowires. Even larger GeTe octahedra of several microns were obtained from a vapor transport process. ${ }^{[49]}$

TEM investigations (Fig. 2) of the octahedral shaped GeTe particles confirmed the unimodal size distribution and edge length of $300 \mathrm{~nm}$ as-found by SEM and revealed all particles to be crystalline.

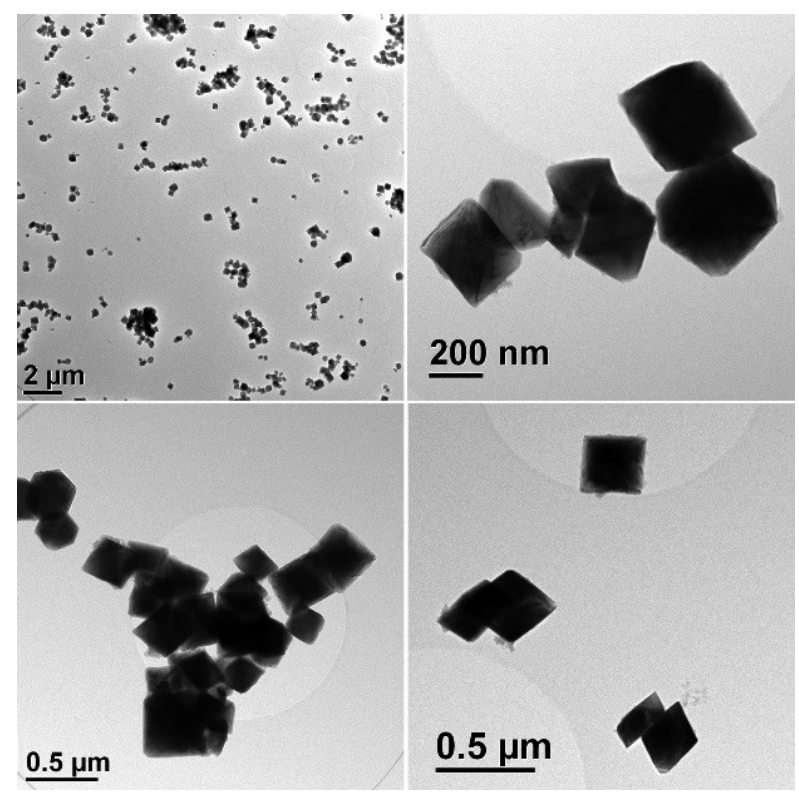

Figure 2. TEM images of GeTe octahedra as-obtained at $160{ }^{\circ} \mathrm{C}$ in oleylamine.

The composition of the GeTe octahedra from EDS nano beam analysis of 20 different crystals was determined to a Ge:Te ratio of 48:52 in average with a standard deviation of 2 for both elements. This agrees, within experimental error, with the expected composition of GeTe. ED patterns from different zone axis orien- tations (given in SI) can be indexed consistently with a rhombohedral cell yielding lattice parameters $a: 5.98 \AA$ and $\alpha: 88.3^{\circ}$, which are in good agreement with $\alpha$-GeTe and with the results from XRD powder diffraction of this sample as shown in figure 5 . It is more convenient to describe $\alpha$-GeTe with the rhombohedral cell in space group $R 3 m$ (No. 160) than with a hexagonal $R$ cell to show the close relationship to the rock salt structure. Figure 3 shows a GeTe crystal viewed along the [100] zone axis with the corresponding ED pattern in the same orientation. Obviously, the corners of the pseudo-octahedra grow in $<100>$ and the edges are in $\langle 110\rangle$ direction. The triangular faces are in $<111\rangle$ direction (cf. Supporting Information). The growing directions are the same as for octahedra of fcc crystals. Parallel lines indicate the $\{002\}$ lattice fringes with a d-value of $293 \mathrm{pm}$ in the HRTEM image. The slight distortion from the cubic symmetry is reflected by the angle of $88.3^{\circ}$ between the $\{002\}$ lattice fringes.

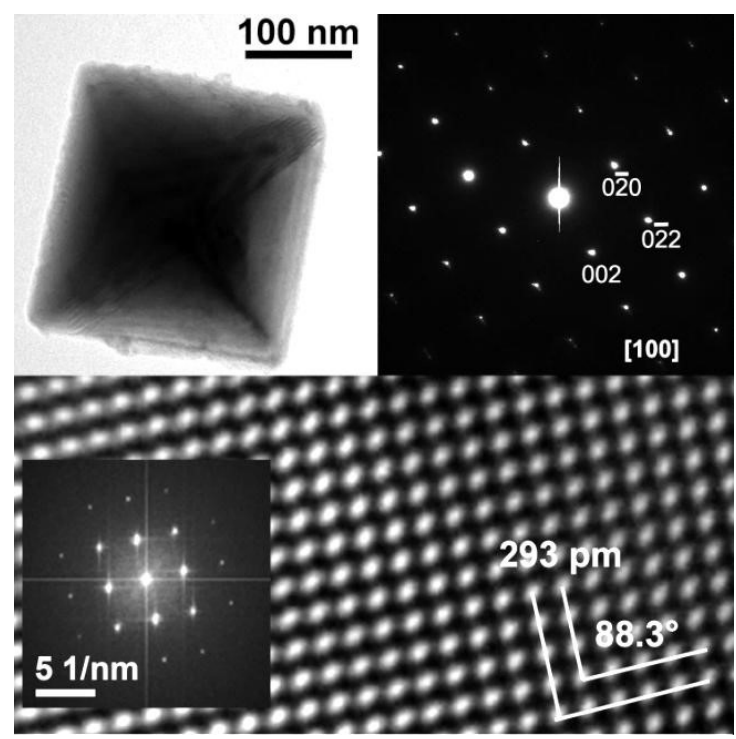

Figure 3. Top: TEM bright field image of an GeTe octahedron in [100] orientation and corresponding ED-pattern. Bottom: HRTEM image and corresponding FT as inset. GeTe as-obtained at $160{ }^{\circ} \mathrm{C}$ in oleylamine.

Our TEM results are in excellent agreement with the findings of Kim et $\mathrm{al}^{[41]}$ on octahedral shaped GeTe nano-crystals. They described their particles to have a unimodal distribution (average value $337 \mathrm{~nm}$ ) and a composition of 47:53 and 56.6:43.4 Ge:Te ratios from two EDS measurements. HRTEM exhibits wellresolved 2D lattice fringes with plane spacings of $222 \mathrm{pm}$, that correspond to lattice planes of $\{024\}$ in hexagonal $R$ GeTe. These findings are within the error of ED and consistent with the d-value of $\{022\}$ fringes in rhombohedral setting as observed in our samples. Recently, Buck et al. reported on the polymer-assisted synthesis of GeTe nanoparticles of octahedral facetted shape. ${ }^{[42]}$ The lattice parameters of the rhombohedral GeTe nanoparticles were proven by ring diffraction patterns from SAED of a number of crystals. The growing direction was deduced from HRTEM to be $<111>$ for the octahedral planes and the composition as derived from EDS was found to be $\mathrm{Ge}_{52} \mathrm{Te}_{48}$. 


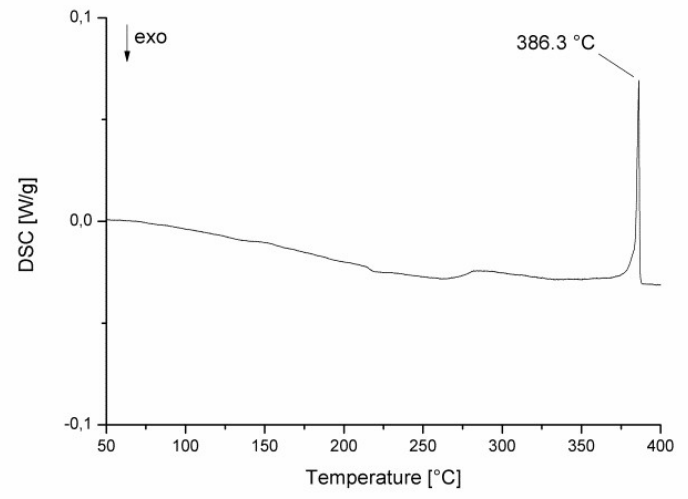

Figure 4. DSC curve of freshly prepared GeTe octahedra.

The TGA/DTA analysis (Fig. $3 \mathrm{SI}$ ) of the isolated GeTe octahedra as-obtained from the reaction in oleylamine at $160{ }^{\circ} \mathrm{C}$ showed an endothermic peak at $716{ }^{\circ} \mathrm{C}$, which is slightly lower than that reported by $\mathrm{Kim}$ et al $\left(721.8^{\circ}\right),{ }^{[41]}$ whereas the melting point of bulk GeTe is significantly higher $\left(725^{\circ} \mathrm{C}\right) .{ }^{[50]} \mathrm{A}$ TGA experiment showed a weight loss of only $1.2 \%$ up to a temperature of $500{ }^{\circ} \mathrm{C}$, which is a good indicator for the purity of the GeTe microcrystals. More substantial weight loss started at 600 ${ }^{\circ} \mathrm{C}$ due to sublimation of GeTe. A DSC study (Fig. 4) showed a first-order solid-solid phase transformation at $386{ }^{\circ} \mathrm{C}$, which is in between the temperatures previously reported $\left(350{ }^{\circ} \mathrm{C},{ }^{[15]} 402\right.$ ${ }^{\circ} \mathrm{C}^{[51]}$ ).

The powder XRD diffraction pattern (Fig. 5) of GeTe obtained at $160{ }^{\circ} \mathrm{C}$ in oleylamine corresponds very well to nanocrystalline $\alpha$-GeTe in space group $R 3 m$. The calculated lattice parameters ( $a$ $=8.3642$ (1) $\AA, c=10.6524$ (3) $\AA, V=645.40$ (3) $\AA^{3}$ ) agree very well with those obtained from the TEM studies as well as the results of the single crystal X-ray structure analysis. The data are also in very good agreement with JCPDS No. 47-1079. ${ }^{[52]}$ The calculated density of the unit cell $\left(6,18 \mathrm{~g} / \mathrm{cm}^{3}\right)$ agrees well with that of bulk $\alpha$-GeTe $\left(6,14 \mathrm{~g} / \mathrm{cm}^{3}\right)$. The determined crystallite size (using the Scherrer equation) of $160 \mathrm{~nm}$ confirms the nanostructure of the produced GeTe particles and corresponds to the values of the TEM studies. In addition, microstrain $(\varepsilon=0.07 \%)$ as another influent effect on the peak broadening in diffractogram was also detected ("Williamson-Hall plot", SI). The calculated texture-coefficients (relationship defined by Barret and Massalski) ${ }^{[66]}$ for the first $11 \mathrm{hkl}$ values did not show any sign of texture effects (SI).

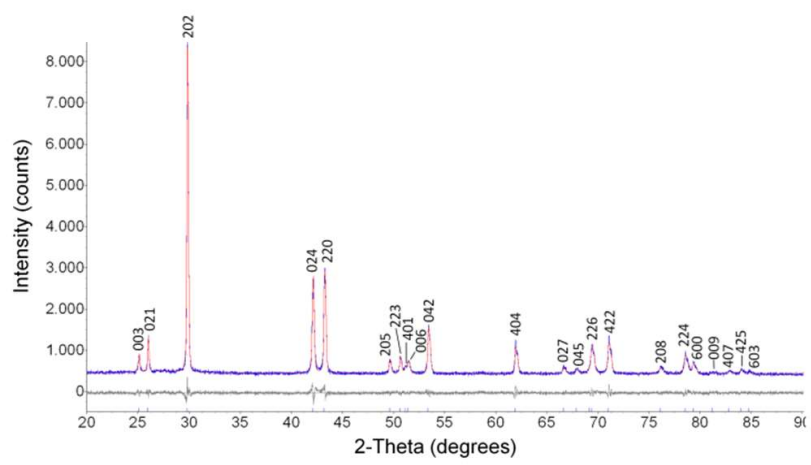

Figure 5. XRD pattern of GeTe nanoparticles formed at $150{ }^{\circ} \mathrm{C}$ after $4 \mathrm{~h}$ including the results from Rietfeld refinement as difference plot $\left(R_{w} p=5.02\right)$.
Since the results of the reaction of $\mathrm{GeCl}_{2}$ dioxane and $\mathrm{Te}\left(\mathrm{SiEt}_{3}\right)_{2}$ in oleylamine were much more promising than those in DIPB, we focused on this reaction and particularly investigated the role of reaction time, reaction temperature and solvent in detail.
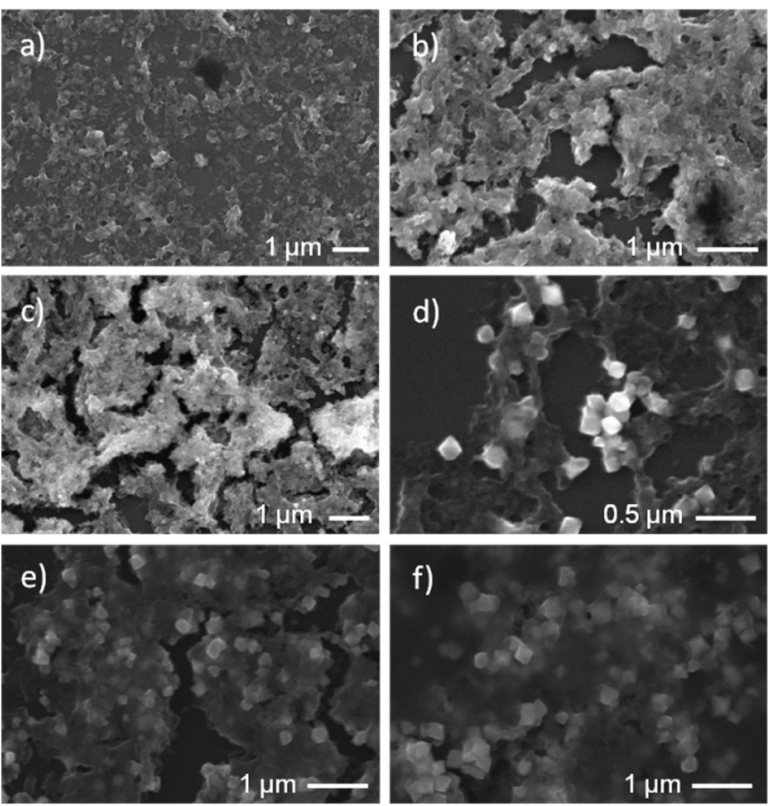

Figure 6. SEM micrographs of time-dependent formation of GeTe octahedra at $150^{\circ} \mathrm{C}$ in oleylamine after a) $5 \mathrm{~min}$, b) $15 \mathrm{~min}$, c) $30 \mathrm{~min}$, d) $60 \mathrm{~min}$, e) $120 \mathrm{~min}$, and f) $240 \mathrm{~min}$.

Growth Mechanism. Reactions between $\mathrm{GeCl}_{2} \cdot$ dioxane and $\mathrm{Te}\left(\mathrm{SiEt}_{3}\right)_{2}$ at $150{ }^{\circ} \mathrm{C}$ in oleylamine were carried out to determine the starting point of crystallization and formation of octahedral GeTe. Figure 6 shows SEM images of the solid precipitate obtained after different reaction times, which show the formation of crystalline GeTe octahedra $60 \mathrm{~min}$ after starting the reaction, whereas shorter reaction times produced particles without defined shape, most likely much smaller particles whose shape couldn't be resolved precisely. These findings point to a thermodynamicallydriven Ostwald-ripening-type crystal growth process, in which large numbers of small GeTe crystals of inhomogeneous shape over time grow to fewer larger crystals with well-defined morphology and shape in order to decrease their energy. The formation of a large number of small GeTe crystals requires a spontaneous precipitation of crystal seeds by a very fast reaction between $\mathrm{GeCl}_{2}$.dioxane and $\mathrm{Te}\left(\mathrm{SiEt}_{3}\right)_{2}$, hence producing a large number of crystal seeds, which is very likely under these specific conditions according to our NMR studies.

Role of Reaction Temperature. The reaction temperature was lowered to $100{ }^{\circ} \mathrm{C}$ in order to verify its influence on the size, shape and crystallinity of the GeTe particles. The formation of octahedral GeTe particles was observed starting at $140{ }^{\circ} \mathrm{C}$ (Fig. 7 ), whereas lower reaction temperatures only gave materials with unspecific morphologies. XRD studies proved that the formation of crystalline GeTe particles started at $140{ }^{\circ} \mathrm{C}$. The size of the crystalline GeTe particles formed at $160{ }^{\circ} \mathrm{C}$ is larger than that of the particles formed at $140{ }^{\circ} \mathrm{C}$ as can be derived from the sharper reflexes. Reactions performed at $180^{\circ} \mathrm{C}$ also gave GeTe particles, whose size and shape were comparable to those obtained at 160 ${ }^{\circ} \mathrm{C}$. These findings somehow correlate with those of previous studies, in which the amorphous-to-crystalline phase transition for $\mathrm{GeTe}$ thin films was found to occur at $145^{\circ} \mathrm{C},{ }^{[53,54]}$ whereas bulk GeTe started to crystallize at roughly $180{ }^{\circ} \mathrm{C} \cdot .^{[55]}$ However, this finding has to be considered as rather coincidentally since the 
solution-based growth of crystalline GeTe particles kinetically differs from the reorganization of solid GeTe from the meta-stable amorphous state to the crystalline state.

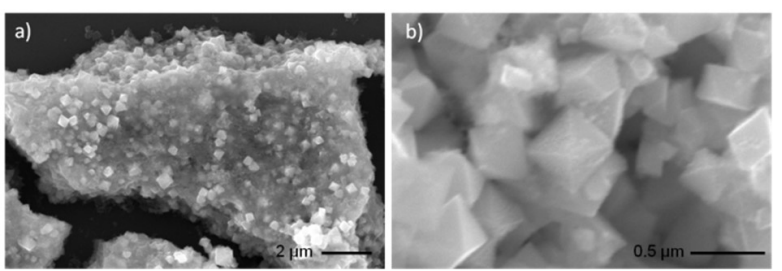

Figure 7. SEM micrographs showing the formation of GeTe octahedra at a) $140{ }^{\circ} \mathrm{C}$ and b) $150{ }^{\circ} \mathrm{C}$.

Role of $\mathbf{T e}\left(\mathrm{SiEt}_{3}\right)_{2}$ Concentration. The reaction of an excess of $\mathrm{Te}\left(\mathrm{SiEt}_{3}\right)_{2}$ with $\mathrm{GeCl}_{2}$-dioxane at $140{ }^{\circ} \mathrm{C}$ in oleylamine proceeded with formation of $\alpha$-GeTe (JCPDS Card No. 47-1079) and crystalline hexagonal Te (JCPDS Card No. 36-1452) according to powder X-ray diffraction studies (Fig. 8).

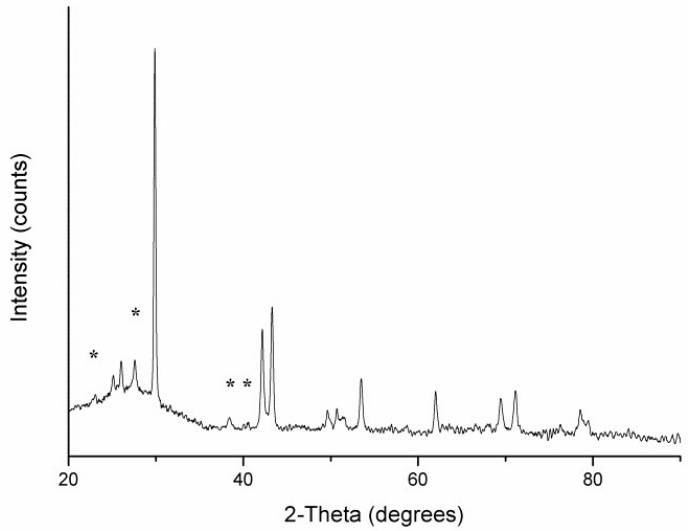

Figure 8. XRD pattern of Te-covered GeTe particles. $\left(^{*}\right)$-labeled reflexes correspond to trigonal Te (P3 21 (No. 152), JCPDS No. 36-1452)

TEM studies revealed the formation of GeTe octahedra, whose surface-is covered with Te nanowires (Fig. 9a, 9b). Te-decorated GeTe particles were previously obtained by Korgel et al. in reactions of diphenyl germane $\mathrm{GePh}_{2}$ with TOPTe in supercritical hexane at $460{ }^{\circ} \mathrm{C}$ in the presence of octanol, oleic acid or isoprene ${ }^{[30]}$ whereas hexadecanethiol completely quenched the Te nanowire formation. The formation of the Te@GeTe particles was explained by initial formation of the GeTe core, from which the Te nanowires grew off their faceted surfaces. Even though the exact mechanism of the Te nanowire formation remained unclear, the authors suggested that the additives either modify the reactant decomposition kinetics or influence the reactivity of the particle surfaces due to the binding/passivation with different capping agents. However, we observed, that the Te wires can be removed by washing the Te@GeTe particles with TOP, resulting in the formation of TOPTe (Fig. 9 c, 9d).

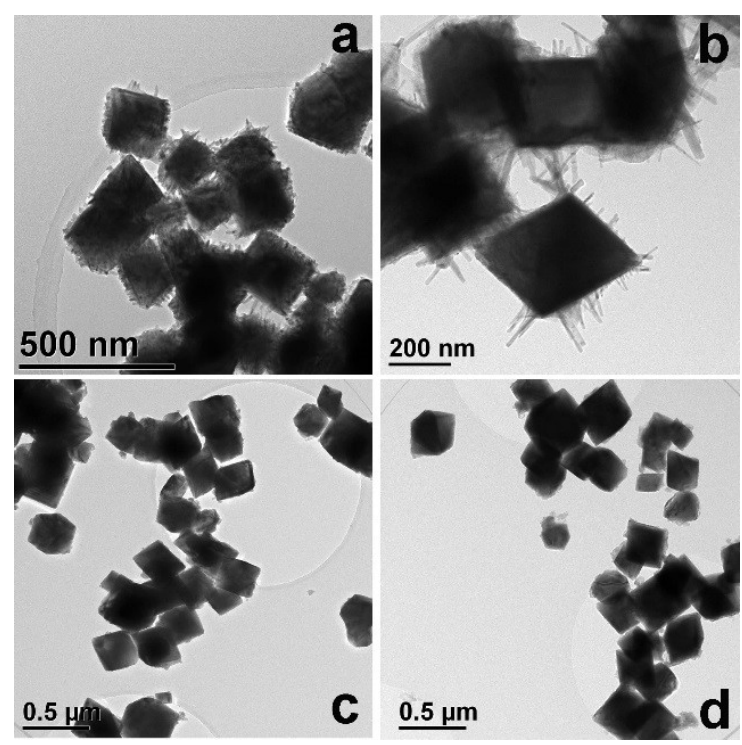

Figure 9. TEM micrographs of GeTe octahedra decorated with elemental Te nanowires $(a, b)$ and after washing with TOP $(c, d)$.

Role of solvent. In order to verify the specific role of the solvent, the reaction of $\mathrm{GeCl}_{2} \cdot$ dioxane and $\mathrm{Te}\left(\mathrm{SiEt}_{3}\right)_{2}$ was further investigated in hexadecylamine, di-n-octylamine, tri-noctylamine, and 1-hexadecanethiol, respectively.
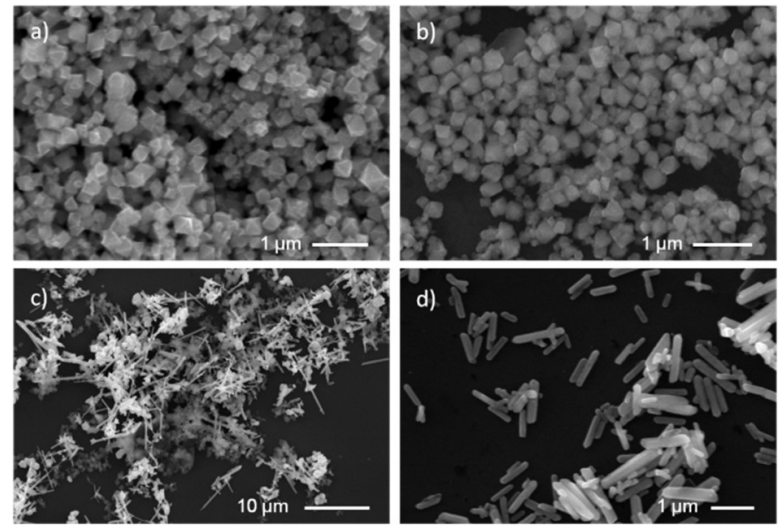

Figure 10. SEM micrographs of Te and GeTe particles as-formed in hexadecylamine (a), di-n-octylamine (b), tri-n-octylamine (c), and 1-hexadecanethiol (d).

Crystalline GeTe particles of octahedral shape were obtained in hexadecylamine and di-n-octylamine, whereas the reactions in trin-octylamine and 1-hexadecanethiol preferably occurred with formation of elemental tellurium (Fig. 10). In particular the formation of Te crystallites in the presence of hexadecanethiol is somewhat surprising, since Milliron et al. reported on the synthesis of GeTe nanoparticles with controlled size and morphology only in the presence of dodecanethiol, ${ }^{[23]}$ while Korgel et al. reported that hexadecanethiol quenches the formation of elemental Te nanowires. ${ }^{[30]}$ These findings also demonstrate that the initial formation of $\mathrm{Te}$ polyanions by amination of $\mathrm{Te}\left(\mathrm{SiEt}_{3}\right) 2$, which only proceeds with primary and secondary amines, seems to play a key role in the reaction mechanism of the GeTe particle formation. In summary, we believe that protic, strongly coordinating solvents are essential for the formation of GeTe nanoparticles with defined size and morphology.

Single crystal analysis. Bulk GeTe has long served as a prototype for phase-change materials as a result of its simple composi- 
tion and structure. ${ }^{[53,56]}$ Amorphous GeTe is a semiconductor with a band gap of $0.8 \mathrm{eV}$ and an electrical resistivity of $10^{3} \Omega \mathrm{cm}$, whereas both values changes dramatically upon crystallization $\left(0.1 \mathrm{eV}, 10^{-4} \Omega \mathrm{cm} \cdot{ }^{[57]}\right.$ As a consequence, the structure of GeTe has been largely investigated in the past. Amorphous GeTe becomes crystalline at roughly $180{ }^{\circ} \mathrm{C}$. A rhombohedral GeTe phase, $\alpha$ $\mathrm{GeTe}$, is formed, which further undergoes a phase transition to the more symmetric high-temperature rock-salt (NaCl-type) phase, which is stable only above $400{ }^{\circ} \mathrm{C}$. The phase-transition was found to occur at $350{ }^{\circ} \mathrm{C},{ }^{[53]}$ in other reports a transition temperature of roughly $400{ }^{\circ} \mathrm{C}$ is reported. ${ }^{[51]}$ However, the structure reports are contradictory. There is little doubt that the structure of $\alpha$-GeTe is related to its high temperature modification $\beta$-GeTe which crystallizes in the $\mathrm{NaCl}$ type of structure. Upon cooling a distortion along the threefold axis occurs and the crystal system undergoes a transition from cubic to rhombohedral. For the rhombohedral modification structure solutions have been reported in the acentric space group $R 3 m$ with (hexagonal) lattice parameters of $a=4.2 \AA$ and $c=10.7 \AA .^{[58,59]}$ However, also reports of a unit cell with a 4-times larger volume and double $a$-axis $(a=8.3 \AA$ and $c=10.7 \AA)^{[60,61]}$ can be found and the structure has been solved in the centric space group $R-3 m^{[62-65]}$ as well as in its acentric counterpart $R 3 m .^{[58]}$

Single GeTe crystals were grown from freshly prepared GeTe upon slow sublimation at $650^{\circ} \mathrm{C}$ and $10^{-3}$ mbar. Crystals of almost perfect octahedral morphology up to $200 \mu \mathrm{m}$ in size were obtained within $16 \mathrm{~h}$ (Fig. 11).
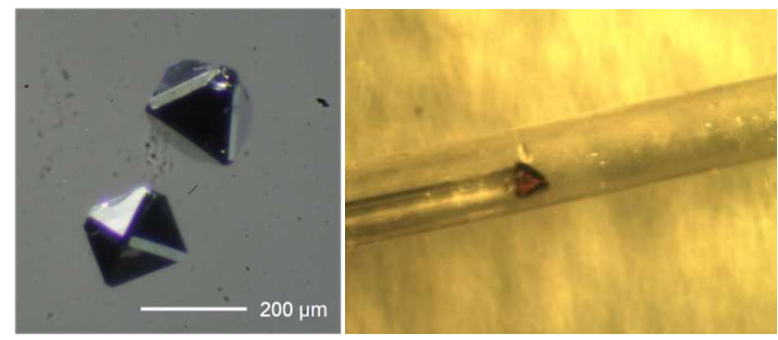

Figure 11. Picture of GeTe single crystals as obtained by slow sublimation (left) and the crystal used for X-ray analysis (right).

Single X-ray structure analysis at low and room temperature confirmed the larger cell with $a=8.471(5) \AA$ and $c=10.392(6)$ $\AA$, which yields a $c / a$ ratio of 1.226 and a Volume of $V=645.8(7)$ $\AA^{3}$ at $80(2) \mathrm{K}$ and $a=8.396(2) \AA$ and $c=10.637(3) \AA$ with a $c / a$ ratio of 1.267 at 298(2) K with a volume of $V=649.43$ (2) $\AA^{3}$ (for details on the data collection and structure solution see Supporting Information). Based on the appearance of weak reflections the smaller cell with half the $a$-axis had to be dismissed. Also a structure solution in the smaller unit cell was unstable and comparison of the calculated powder pattern based on this solution and the recorded powder pattern clearly rule out the smaller unit cell (Fig. 5 and SI).

Structure solution in the larger unit cell succeeded both in the acentric space group $R 3 m$ and the centric space group $R-3 m$. The $R$-values as well as the goodness of fit for a structure solution as an inversion twin in the acentric space group were noticeably smaller. A clear proof why a structure report in the acentric space group should be preferred over the centric becomes clear when analyzing the anisotropic displacement parameters (Fig. 12).
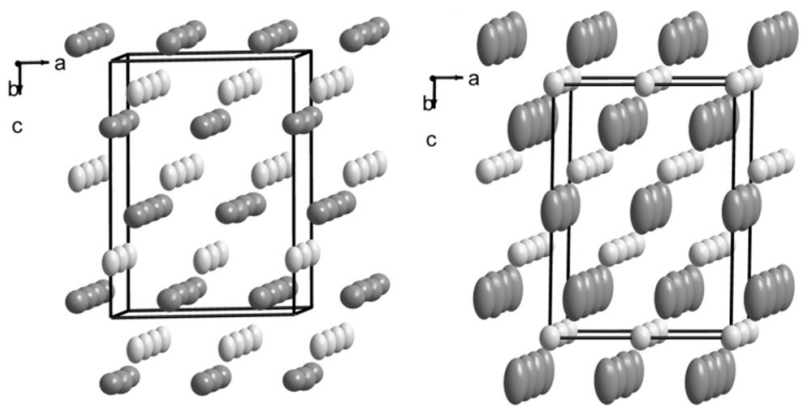

Figure 12. Representations of the structure solution of $\alpha-\mathrm{GeTe}$ with $a=8.396(2) \AA$ and $c=10.637(3)$ in $R 3 m$ (left) and $R-3 m$ (right). The displacement ellipsoids are plotted at a $90 \%$ probability.

Conclusions. Nanosized GeTe octahedra were obtained from the reaction of $\mathrm{GeCl}_{2} \cdot \mathrm{dioxane}$ and $\mathrm{Te}\left(\mathrm{SiEt}_{3}\right)_{2}$ at temperatures as low as $160^{\circ} \mathrm{C}$. The solvent was found to play a critical role on the reaction mechanism, since highly stoichiometric GeTe octahedra were only obtained in the presence of primary and secondary amines. Oleylamine has to be considered rather as reagent, which strongly affects the chemistry of the whole reaction, than as simple capping agent. In contrast, reactions in DIPB occurred with elimination of $\mathrm{Et}_{3} \mathrm{SiCl}$ and subsequent formation of agglomerated GeTe particles, whereas reactions in tri-n-octylamine and 1hexadecanethiol produced elemental tellurium. The presence of protic, strongly coordinating solvents is essential for the formation of GeTe particles with defined chemical composition, size and shape. In the presence of an excess of $\mathrm{Te}\left(\mathrm{SiEt}_{3}\right)_{2}, \mathrm{GeTe}$ particles decorated with Te nanowires were obtained. The Te nanowires can be removed by washing the particles with TOP. A single crystal and powder X-ray determination proved that GeTe crystallizes in the acentric space group R3m.

\section{ASSOCIATED CONTENT}

\section{Supporting Information}

CIF files giving crystallographic data for GeTe as well as details on the in situ 1H NMR, TGA and TEM studies are given in the Supporting Information File. This material is available free of charge via the Internet at http://pubs.acs.org.

\section{AUTHOR INFORMATION}

\section{Corresponding Author}

* To whom correspondence should be addressed. E-Mail: stephan.schulz@uni-due.de.

\section{Author Contributions}

All authors have given approval to the final version of the manuscript.

\section{ACKNOWLEDGMENT}

StS and AVM would like to thank the Mercator Foundation for support in the project SMILE.

\section{ABBREVIATIONS}

ALD, atomic layer deposition; DIPB, 1,3-diisopropyl benzene; $\mathrm{ED}$, electron diffraction; EDX, energy-dispersive X-ray spectroscopy; SAED, selected area electron diffraction; SEM, scanning electron microscopy; TEM, transmission electron microscopy; TGA, thermogravimetric analysis; TOP, tri-n-octylphosphine, TOPO, tri-n-octylphosphine oxid; XRD, X-ray diffraction. 


\section{REFERENCES}

(1) For a recent review article see: Gao, M.-R.; Xu, Y.-F.; Jiang, J.; Yu, S.-H. Chem. Soc. Rev. 2013, 42, 2986-3017.

(2) Welnic, W.; Botti, S.; Reining, L.; Wuttig, M. Phys. Rev. Lett. 2007, 98, 236403-1-4.

(3) Welnic, W.; Pamungkas, A.; Detemple, R.; Steimer, C.; Blügel, S.; Wuttig, M. Nat. Mater. 2006, 5, 56-62.

(4) Wuttig, M.; Lüsebrink, D.; Wamwangi, D.; Wenic, W.; Gilleßen, M.; Dronskowski, R. Nat. Mater. 2007, 6, 122-128.

(5) Lencer, D.; Salinga, M.; Wuttig, M. Adv. Mater. 2011, 23, 20302058.

(6) See also: Phase Change Materials: Science and Application; Raoux, S.; Wuttig, M., Eds.; Springer: New York, 2009

(7) Wuttig, M.; Yamada, N. Nat. Mater. 2007, 6, 824-832.

(8) Snyder, J.; Toberer, E. S. Nat. Mater. 2008, 7, 105-114.

(9) Raoux, S.; Welnic W.; Ielmini, D. Chem. Rev. 2010, 110, 240-267.

(10) Polking, M. J.; Han, M.-G.; Yourdkhani, A.; Petkov, V.; Kisie-

lowski, C. F.; Volkov, V. V.; Zhu, Y.; Caruntu, G.; Alivisatos, A. P.; Ramesh, R. Nat. Mater. 2012, 11, 700-709.

(11) Caldwell, M. A.; Jeyasingh, R. G. D.; Wong, H.-S. P.; Milliron, D. J. Nanoscale 2012, 4, 4382-4392.

(12) Xiao, G.; Wang, Y.; Ning, J.; Wei, Y.; Liu, B.; Yu, W. W.; Zou, G.; Zou, B. $R S C A d v$. 2013, 3, 8104-8130.

(13) Bruns, G.; Merkelbach, P.; Schlockermann, C.; Salinga, M.; Wuttig, M.; Happ, T. D.; Philipp, J. B.; Kund, M. Appl. Phys. Lett. 2009, 95, 043108-1-3.

(14) Lankhorst, M. H. R.; Ketelaars, B.; Wolters, R. A. M. Nat. Mater. 2005, 4, 347-352.

(15) Steigmeier, E. F.; Harbeke, G. Solid State Commun. 1970, 8, 1275-1279.

(16) Wood, C. Rep. Prog. Phys. 1988, 51, 459-539.

(17) Raoux, S.; Rettner, C. T.; Jordan-Sweet, J. L.; Kellock, A. J.; Topuria, T.; Rice, P. M.; Miller, D. C. J. Appl. Phys. 2007, 102, 094305-1-8.

(18) Zhang, Y.; Raoux, S.; Krebs, D.; Krupp, L. E.; Topuria, T.; Caldwell, M. A.; Milliron, D. J.; Kellock, A.; Rice, P. M.; Jordan-Sweet, J. L.; Wong, H.-S. P. J. Appl. Phys. 2008, 104, 074312-1-5.

(19) Martens, H. C. F.; Vlutters, R.; Prangsma, J. C. J. Appl. Phys. 2004, 95, 3977-3983.

(20) Wang, W. J.; Shi, L. P.; Zhao, R.; Lim, K. G.; Lee, H. K.; Chong, T. C.; Wu, Y. H. Appl. Phys. Lett. 2008, 93, 043121-1-3.

(21) Raoux, S.; Shelby, R. M.; Jordan-Sweet, J. L.; Munoz, B.; Salinga, M.; Chen, Y.-C.; Shih, Y.-H.; Lai, E.-K.; Lee, M.-H. Microelectron. Eng. 2008, 85, 2330-2333.

(22) Sun, X.; Yu, B.; Ng, G.; Meyyappan, M. J. Phys. Chem. C 2007, $111,2421-2425$

(23) Caldwell, M. A.; Raoux, S.; Wang, R. Y.; Wong, H. S. P.; Milliron, D. J. J. Mater. Chem. 2010, 20, 1285-1291.

(24) Vaughn II, D. D.; Schaak, R. E. Chem. Soc. Rev. 2013, 42, 28612879 .

(25) Lee, M.-K.; Kim, T. G.; Ju, B.-K.; Sung, Y.-M. Cryst. Growth Des. 2009, 9, 938-941.

(26) Jennings, A. T.; Jung, Y.; Engel, J.; Agarwal, R. J. Phys. Chem. C 2009 113, 6898-6901.

(27) Arachchige, I. U.; Soriano, R.; Malliakas, C. D.; Ivanov, S. A.; Kanatzidis, M. C. Adv. Funct. Mater. 2011, 21, 2737-2743.

(28) Polking, M. J.; Urban, J. J.; Milliron, D. J.; Zheng, H.; Chan, E.; Caldwell, M. A.; Raoux, S.; Kisielowski, C. F.; Ager, J. W.; Ramesh, R.; Alivisatos, A. P. Nano Lett. 2011, 11, 1147-1152.

(29) Polking, M. J.; Zheng, H. M.; Ramesh, R.; Alivisatos A. P., J. Am. Chem. Soc. 2011, 133, 2044-2047.

(30) Tuan, H. Y.; Korgel, B. A. Cryst. Growth Des. 2008, 8, 25552561

(31) Buck, M. R.; Sines, I. T.; Schaak, R. E. Chem. Mater. 2010, 22, 3236-3240.

(32) Murray, C. B.; Norris, D. J.; Bawendi, M. G. J. Am. Chem. Soc. 1993, $115,8706-8715$.

(33) Schulz, S.; Andruh, M.; Pape, T.; Heinze, T.; Roesky, H. W.; Häming, L.; Kuhn, A.; Herbst-Irmer, R. Organometallics 1994, 13, 40044007 .
(34) Corrigan, J. F.; Fenske, D. Angew. Chem. Int. Ed. 1997, 36, 19811983.

(35) DeGroot, M. W.; Cockburn, M. W.; Workentin, M. S.; Corrigan; J. F. Inorg. Chem. 2001, 40, 4678-4685.

(36) Dehnen, S.; Eichhöfer, A.; Fenske, D. Eur. J. Inorg. Chem. 2002, $2,279-317$.

(37 ) For a review article see: Dehnen, S.; Eichhöfer, A.; Corrigan, J. F.; Fenske, D. In Nanoparticles - From Theory Applications, Ed. Schmid, G., 2nd ed., Wiley, Weinheim, 2010

(38) Pore, V.; Hatanpää, T.; Ritala, M.; Leskelä, M. J. Am. Chem. Soc. 2009, 131, 3478-3480.

(39) Pore, V.; Knapas, K.; Hatanpää, T.; Sarnet, T.; Kemell, M.; Ritala, M.; Leskelä, M.; Mizohata, K. Chem. Mater. 2011, 23, 247-254.

(40) Zastrow, S.; Gooth, J.; Boehnert, T.; Heiderich, S.; Toellner, W.; Heimann, S.; Schulz, S.; Nielsch, K. Semicond. Sci. Technol. 2013, 28, 035010-1-6.

(41) Kim, M. H.; Gupta, G.; Kim, J. RSC Adv. 2013, 3, 288-292.

(42) Buck, M. R.; Biacchi, A. J.; Popczun, E. J.; Schaak, R. E., Chem. Mater. 2013, 25, 2163-2171.

(43) Detty, M. R.; Seidler, M. D. J. Org. Chem. 1982, 47, 1354-1356.

(44) Sheldrick, G. M. Acta Crystallogr. Sect. A 1990, 46, 467-473.

(45) Sheldrick, G. M., SHELXL-97, Program for the Refinement of Crystal Structures University of Göttingen, Göttingen (Germany) 1997 (see also: Sheldrick, G. M. Acta Crystallogr. Sect. A 2008, 64, 112-122.).

(46) Knapas, K.; Hatanpää, T.; Ritala, M.; Leskelä, M. Chem. Mater. 2010, 22, 1386-1391.

(47) Mourdikoudis, S.; Liz-Marzán, L. M. Chem. Mater. 2013, 25, 1465-1476.

(48) Hollemann Wiberg, Lehrbuch der Anorganischen Chemie, 102. Auflage, deGruyter, Berlin, 2007.

(49) Chung, H.-S.; Jung, Y.; Kim, S. C.; Kim, D. H.; Oh, K. H.; Agarwal, R. Nano Lett. 2009, 9, 2395-2401.

(50) Shevchik, N. J.; Tejeda, J.; Langer, D. W.; Cardona, M. Phys. Rev. Lett. 1973, 30, 659-662.

(51) Tomaszewski, P. E. in Structural phase transitions in crystals. I. Database, Phase Transitions: A Multinational Journal, 1992, 38:3, 127220, DOI:10.1080/01411599208222899.

(52) Grier, D.; McCarthy, G.; Seidler, D.; Boudjouk, P.; North Dakota State Univ., Fargo, ND, USA, ICDD Grant-in-Aid, (1993) JCPDS 00-0471047.

(53) Chopra, K. L.; Bahl, S. K. J. Appl. Phys. 1969, 40, 4171-4178.

(54) Andrikopoulos, K. S.; Yannopoulos, S. N.; Voyiatzis, G. A.; Kolobov, A. V.; Ribes, M.; Tominaga, J. J. Phys. Condens. Matter. 2006, $18,965-979$.

(55) Gervacio Arciniega, J. J.; Prokhorov, E.; Espinoza Beltran, F. J.; Trapaga, G. (2012). Crystallization of Ge:Sb:Te Thin Films for Phase Change Memory Application, Crystallization - Science and Technology, Dr. Marcello Andreeta (Ed.), InTech, DOI: 10.5772/35577. Available from: http://www.intechopen.com/books/crystallization-science-andtechnology/crystallization-of-gesbte-thin-films-for-phase-change-memory.

(56) Bahl, S. K.; Chopra, K. L. J. Appl. Phys. 1969, 40, 4940-4947.

(57) Bahl, S. K.; Chopra, K. L. J. Appl. Phys. 1970, 41, 2196-2212.

(58) Chattopadhyay, T.; Boucherle, J. X.; von Schnering, H. G. J. Phys. C: Solid State Phys. 1987, 20, 1431-1440.

(59) Goldak, J.; Barrett, C. S.; Innes, D.; Youdelis, W. J. Chem. Phys. 1966, 44, 3323-3325.

(60) Matsunaga, T.; Kojima, R.; Yamada, N.; Kifune, K.; Kubota, Y.; Tabata, Y.; Takata, M. Inorg. Chem. 2006, 45, 2235-2241.

(61) Nonaka, T.; Ohbayashi, G.; Toriumi, Y.; Mori, Y.; Hashimoto, H. Thin Solid Films 2000, 370, 258-261.

(62) Wiedemeier, H.; Siemers, P. A. High Temperature Science 1984, 17, 395-408.

(63) Wiedemeier, H.; Siemers, P. A. Z. Anorg. Allg. Chem. 1977, 431, 299-304.

(64) Abrikosov, N. Kh.; Avilov, E. S.; Karpinskii, O. G.; Radkevich, O. V.; Shelimova, L. E. Inorg. Mater. 1984, 201, 20-24.

(65) Parker, S. G.; Pinnell, J. E.; Swink, L. N. J. Mater. Sci. 1974, 9, 1829-1832.

(66) Barret, C.; Massalski, T. B. in Structure of metals. Pergamon Press: Oxford, 1980. 
SYNOPSIS TOC. Crystalline GeTe octahedra were obtained from the reaction of $\mathrm{GeCl}_{2} \cdot \operatorname{dioxane}$ and $\mathrm{Te}\left(\mathrm{SiEt}_{3}\right)_{2}$ in different organic solvents at low temperature and characterized by SEM, TEM, EDX and XRD. The solvents critically influence the reaction mechanism, and oleylamine serves rather as reagent than as a simple capping agent. The role of reaction time, reaction temperature and precursor concentration on the GeTe particle formation is shown. In addition, the single crystal X-ray structure of GeTe is reported.

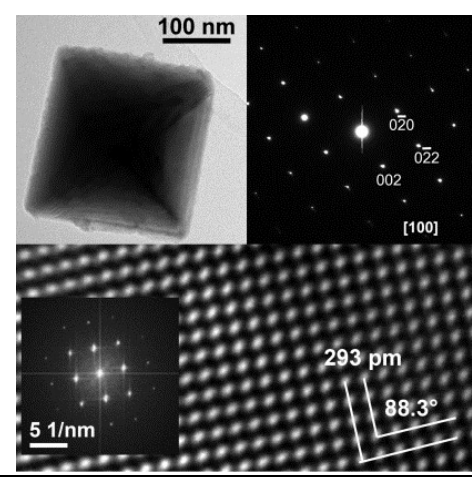




\section{DuEPublico}

This text is made available via DuEPublico, the institutional repository of the University of Duisburg-Essen. This version may eventually differ from another version distributed by a commercial publisher.

DOI: $\quad 10.1021 / \mathrm{ic} 402266 \mathrm{j}$

URN: urn:nbn:de:hbz:464-20201116-113050-4

This document is the Accepted Manuscript version of a Published Work that appeared in final form in: Inorg. Chem. 2013, 52, 24, 14326-14333, copyright (C) American Chemical Society after peer review and technical editing by the publisher.

To access the final edited and published work see: https://doi.org/10.1021/ic402266j

All rights reserved. 